\title{
Duodenal Neuroendocrine Tumor
}

National Cancer Institute

\section{Source}

National Cancer Institute. Duodenal Neuroendocrine Tumor. NCI Thesaurus. Code C135080.

A well differentiated, low or intermediate grade tumor with neuroendocrine differentiation that arises from the duodenum. 